\title{
Creating a hypertext markup language document for an information server
}

\author{
JOSEY Y. M. CHU, WILLIAM L. PALYA, and DONALD E. WALTER \\ Jacksonville State University, Jacksonville, Alabama
}

\begin{abstract}
This paper briefly overviews the World-Wide Web. It then provides a short tutorial on the use of the hypertext markup language to publish information on the web. Hypertext markup language is a special page-layout language that was developed to help make creating and retrieving information on the web consistent and efficient. Hyperlinks within hypertext markup language make the language especially powerful because they enable the browser to transparently retrieve images, movies, or audio files from virtually any computer on earth simply by clicking on an item displayed on the monitor.
\end{abstract}

If one considers the sum total of all the information on all computers on earth, it boggles even the wildest of imaginations. Yet a single idea has led to each of us having easy and productive use of virtually all of the information. This idea was that many of the computers across the globe are connected together much like a network of telephones. Provided that there are some simple protocol standards, any computer can be instructed to "call up" any other computer and intelligently retrieve any desired piece of information. The instantiation of that idea has resulted in an easy-to-use global network of computers commonly known as the Internet and the World-Wide Web. The network has become an almost limitless source of information and venue for publication.

Necessarily, there must be a coherent mechanism for getting and providing information. Figure 1 'illustrates the two basic elements of the network: browsers that are information-receiving programs, and servers that are the information-providing programs. Users wishing to browse through the information provided by various information servers must use a browser program. There are several different browsers available, such as Mosaic, Netscape, MacWeb, Gopher, Cello, and Lynx. Each site wanting to provide information uses an informationserver program. The server receives requests from users on the network and sends requested information. Currently, there are several information-server programs, such as Gopher daemon (Gopherd), hypertext transfers protocol daemon (HTTPd), files transfer protocol daemon (FTPd), and Macintosh hypertext transfer protocol daemon (MacHTTPd).

A browser can be instructed to retrieve a wide variety of information from a server. The information could be text, pictures, audio, or even movies. Figure 1 illustrates some of the details of the process. The user directs the

The authors gratefully acknowledge the contributions of Edmond Venator and Elizabeth Palya. Correspondence and requests for reprints should be sent to W. L. Palya, Department of Psychology, Jacksonville State University, Jacksonville, AL 36265 (e-mail: palya@sebac. jsu.edu). browser (in this case, Mosaic) to retrieve information from some particular directory on a server. The information is downloaded to a buffer on the user's computer. Mosaic reads and interprets this information and then lays out the display of that material on the user's monitor, as directed by the obtained file. The standard protocol is labeled hypertext. It specifies how the text is to be formatted for display on the user's screen by the browser program.

\section{HYPERTEXT MARKUP LANGUAGE}

Hypertext markup language (HTML) is the standard procedure for defining how text, pictures, movies, and sound recordings are to be laid out or presented by a browser such as Mosaic. The rest of this paper provides a short tutorial on using some of the basic HTML commands for people putting information on servers in order to make it available on the Internet. The present tutorial will overview text formatting, picture inclusion, and hyperlinks. Hyperlinks are an easy-to-use method of retrieving additional files from the home server or files from other servers located anywhere on earth.

An actual HTML document is constructed using plain ASCII text with plain ASCII format commands. A complete HTML document could be typed on a manual typewriter. The HTML formatting codes permit any text editor (e.g., teco, vi, or emacs, as well as Word Perfect and Word) and any "dumb terminal" to generate an HTML document. The browser program creates the reader-friendly, artistic, formatted displays by carrying out the formatting as specified in the HTML code. Any browser at any site will lay out the information on the user's monitor as specified by the HTML commands in pretty much the same way.

HTML uses designators called markup tags to specify how the browser is to format and display the information provided in the HTML document. For example, if emboldened text is desired, then $\langle$ EM $>$ text $\langle$ /EM $>$ is entered. When Mosaic encounters the $<E M>$ tag (begin emboldening), it emboldens all the following characters 


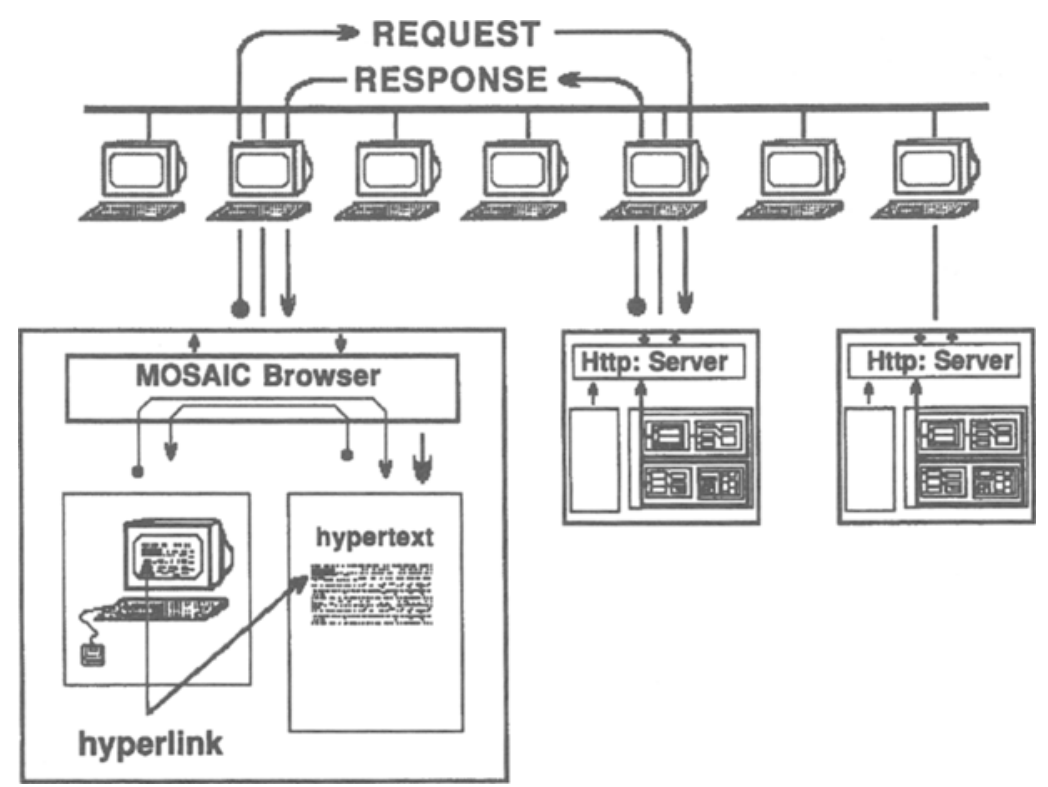

Figure 1. An illustration of a browser-server interaction called hypertinks. Hyperlinks permit users to simply click on a highlighted item that appears on the monitor and thereby retrieve information from (1) a different document on the same server, (2) a document on a different server anywhere on earth, or even (3) a different part of a current document.

until the $</ E M>\operatorname{tag}$ (stop emboldening) is encountered. Typically, HTML tags occur in pairs, and the end tag contains a slash $(/)$ preceding the type of command it terminates.

Browsers ignore the case of the text in the HTML commands. Browsers do preserve the case in the text itself, however. Browsers also ignore carriage returns or extra blanks inserted by the text editor into an HTML document. All formatting of the output of the browser to the user's monitor is standard (consecutive characters are simply put in consecutive position and consecutive lines), unless explicitly specified by the markup tags.

\section{Basic Markup Tags}

Title. The first thing to do when creating an HTML document is to give it a title. Most titles are displayed at the top of the user's screen. In Figure 2, the title of the document is "SEBAC Psychology Server" and is displayed above the pull-down menu.

The following example illustrates how that title would be specified in the HTML document. Note that the title is preceded and followed by the appropriate markup tags $(<$ Title $>$. . $<$ /Title $>)$ :

$$
<\text { Title }>\text { SEBAC Psychology Server </Title }>
$$

Paragraphs. Paragraphs in HTML documents are separated by the paragraph tag $(<\mathrm{P}>)$. When a browser such as Mosaic encounters the paragraph tag, it inserts a line break or line feed before the next sentence (i.e., it starts a new paragraph). Otherwise, it simply appends the text into consecutive character positions and consecutive lines, ignoring multiple blanks and carriage re- turns. The paragraph tag, unlike many other markup tags, is not used in pairs; placing a single paragraph tag at the end of the paragraph is sufficient. For example, the following HTML code:

This is paragraph one text. $<\mathrm{P}>$ This is paragraph two text.

would result in the following browser display:

$$
\begin{aligned}
& \text { This is paragraph one text. } \\
& \text { This is paragraph two text. }
\end{aligned}
$$

Preformatted text. Preformatted text tags ( $<$ PRE $>$... $<$ PRE $>$ ) allow the spaces, new lines, and tabs inserted in the original text by the text editor to be interpreted "as is" by a browser such as Mosaic. All text between the preformatted tags will be displayed precisely as typed (in nonproportional, courier, or typewriter font). For example, the following HTML code:

$<$ PRE $>$
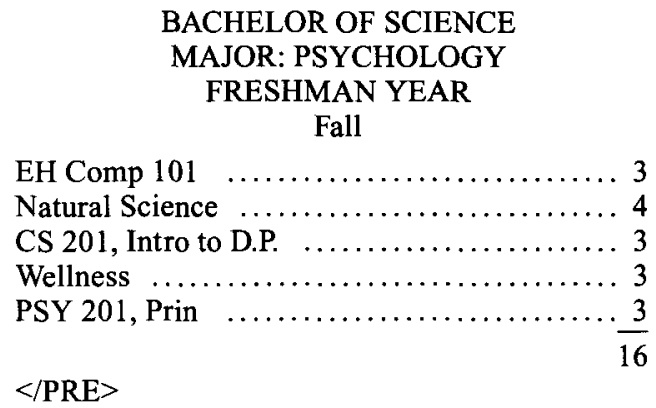

would result in the following browser display: 


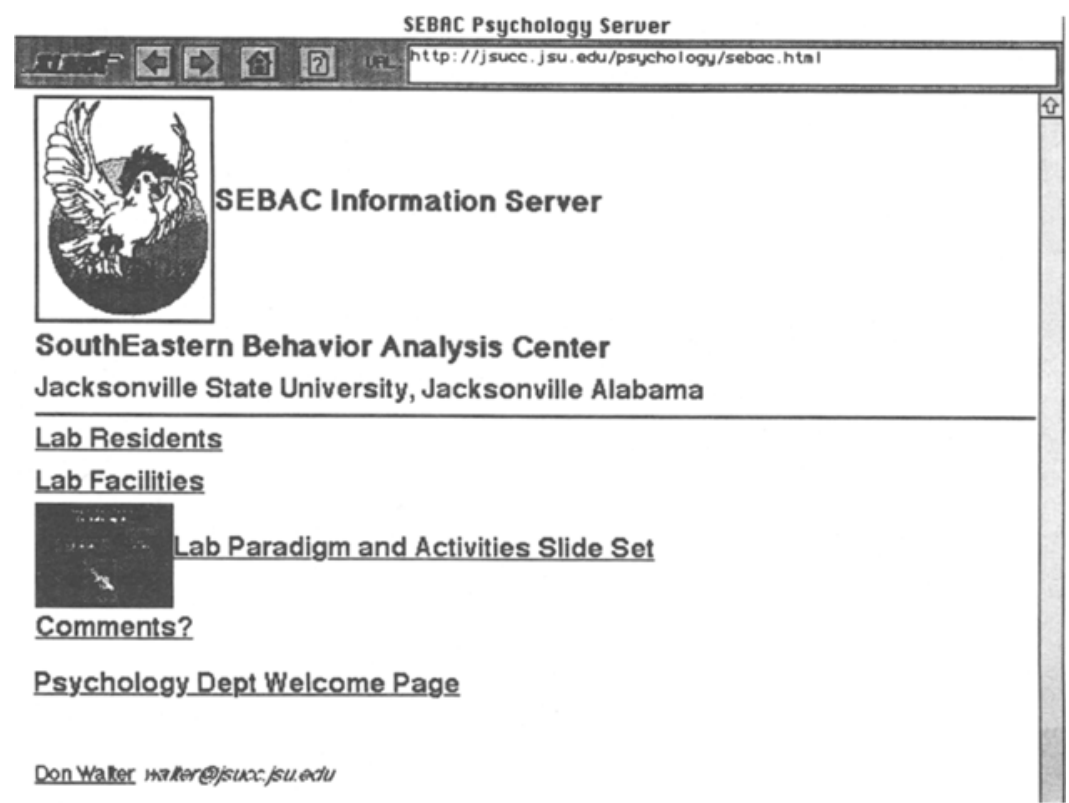

Figure 2. An example of a welcome page (SEBAC welcome page) as displayed by MacWeb on a Macintosh computer retrieved through a URL.

\section{BACHELOR OF SCIENCE \\ MAJOR: PSYCHOLOGY FRESHMAN YEAR \\ Fall}

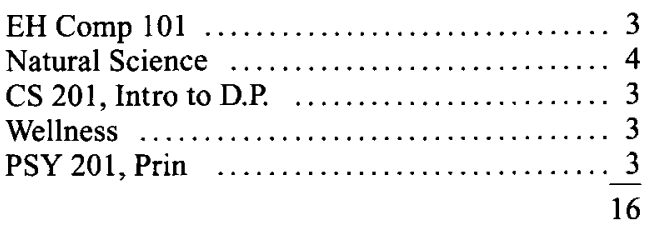

Forced line breaks. The forced-line-break command $(<\mathrm{BR}>)$ forces a line break before subsequent text. Unlike the paragraph tag, which inserts a blank line between sentences to clearly indicate the beginning of a new paragraph, the forced-line-break command does not insert a blank line between text strings. The usage of the line-break command is similar to the paragraph tag in that there is only one end-of-line directive needed. One use for the forced-line-break command is the formatting of an address. For example, the following HTML code:

$$
\begin{gathered}
\text { Southeastern Behavioral Analysis Center }<\text { BR }> \\
\text { Jacksonville State University } \\
<\text { BR }>\text { Department of Psychology }<\text { BR }> \\
\text { Jacksonville, Alabama } 36265<\text { BR }>
\end{gathered}
$$

would result in the following browser display:

$$
\begin{aligned}
& \text { Southeastern Behavioral Analysis Center } \\
& \text { Jacksonville State University } \\
& \text { Department of Psychology } \\
& \text { Jacksonville, Alabama } 36265
\end{aligned}
$$

Horizontal rules. It is sometimes handy to separate items vertically in a display with a simple horizontal line from one side of the display screen to the other. To pro- duce a horizontal line on the browser window, a horizontal rule is used. The horizontal rule tag $(<\mathrm{HR}>)$ produces a horizontal line from the left edge of the screen to the right edge of the screen.

Headings. HTML is able to specify six different levels of headings, designated "Hl" through "H6." Heading levels vary in font size, with $\mathrm{H} 1$ being displayed in the largest font. Headings are normally also rendered in bold fonts by the browser program. An example of the HTML code is:

$\begin{array}{ccccc}<\mathrm{H} 1> & \text { Level } & \text { one } & \text { heading } & </ \mathrm{H} 1> \\ \ldots & \ldots \ldots & \ldots & \ldots \ldots & \ldots \\ \ldots & \ldots \ldots & \ldots \ldots & \ldots \ldots & \ldots \\ \ldots & \ldots \ldots & \ldots \ldots & \ldots \ldots & \ldots \\ \ldots & \ldots \ldots & \ldots \ldots & \ldots \ldots & \ldots \\ <\mathrm{H} 6> & \text { Level } & \text { six } & \text { heading } & </ \mathrm{H} 6>\end{array}$

Unnumbered lists. The code to generate an unnumbered list is $\langle\mathrm{UL}\rangle \ldots</ \mathrm{UL}\rangle$. To enter the items in the list, first the tag command $<\mathrm{UL}>$ is used, which is then followed by the $<\mathrm{LI}>$ item tag before each item. Individual items of the list do not require a closing tag. When the entire list has been completed, the unnumbered list is closed by the $</ \mathrm{UL}>$ command tag. For example, the following HTML code:

$$
\begin{aligned}
& <\mathrm{UL}> \\
& <\mathrm{LI}>\text { item A } \\
& <\mathrm{LI}>\text { item B } \\
& <\text { UUL }>
\end{aligned}
$$

would result in the following browser display:

- item A

- item B 
Numbered lists. A numbered list enumerates each item in the list. The code to generate a numbered list is $<\mathrm{OL}>\ldots</ \mathrm{OL}>$. The $<\mathrm{OL}>$ command tag is followed by the $<\mathrm{LI}>$ list item tag before each item. When the list is completed, the list is closed by the $</ \mathrm{OL}>$ command tag. For example, the following HTML code:

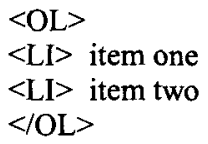

would result in the following browser display:

1. item one

2. item two

Character formatting. HTML has the ability to specify that words or sentences be displayed in special styles by the browser. The following tags are used to enclose the text that is to be presented in a special style. If text is to be emboldened, $\angle \mathrm{B}>\ldots</ \mathrm{B}>$ is used. If the text is to be italicized, $<\mathrm{I}>\ldots</ \mathrm{I}>$ is used. If courier, or text that appears typed with a typewriter, is desired, $<\mathrm{TT}\rangle_{\ldots} .</ \mathrm{TT}>$ is used. For example, the following HTML code:

$$
<\text { I }>\text { this is text }</ \text { I }>
$$

would result in the following browser display:

\section{this is text}

Special characters. There are four special ASCII characters $(<,>, \&$, and ") that are interpreted by browser programs as special commands and, therefore, cannot be used "as is" in an HTML document. The angle brackets are interpreted as the beginning and ending of HTML tags, the ampersand is defined as the beginning of an escape sequence, and the double quote specifies a string of characters. An escape sequence must be used in place of these actual characters to tell the browser program to display these four characters. The escape sequences for these four special characters are:

$\begin{array}{lll}\text { \&lt; } & \ldots \ldots & < \\ \text { \&gt; } & \ldots \ldots & > \\ \text { \&amp; } & \ldots \ldots & \text { \& } \\ \text { \&quot; } & \ldots . & \text { " }\end{array}$

Address tags. When producing information for browsers, the author's name and e-mail address are typically placed at the end of the document. HTML has a special tag that is used for addresses. The display is rendered in italics. For example, the following HTML code:

<Address> Walter@sebac.jsu.edu </Address>

would result in the following browser display:

$$
\text { Walter@sebac.jsu.edu }
$$

\section{Images}

Most browsers are able to display graphic material in either "X Bitmap" (XBM) or "GIF" format. To include an image in the display produced by a browser, the following general form is used in the HTML document:

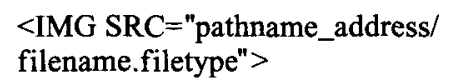

$<$ IMG is the HTML image tag that tells the browser that an image or graphical material will follow. SRC = "..." designates that the pathname/address or source of that image follows. It is enclosed in quotes. Pathname address is the specification of the path name to where the image file can be obtained. Filename specifies the name of the file and filetype specifies the file format standard. > closes the command. For example, to direct Mosaic to display an image named jsulogo.gif (located on the same directory, which eliminates the need for a pathname/address), the following code could be used:

$$
\text { <IMG SRC ="jsulogo.gif"> }
$$

Some World-Wide Web browsers cannot display images. For those browsers, a text alternative to replace the image can be specified in the HTML document. The code ALT $=" .$. " allows those browsers to display text in place of the image. In the following example, the phrase "JSU Logo" will be displayed by text-only browsers:

$$
\text { <IMG SRC ="jsulogo.gif" ALT="JSU Logo"> }
$$

\section{Hyperlinks}

Figure 1 illustrates one of the most powerful and useful features of the browser-server interaction. It is an intelligent information retrieval capability called hyperlink. Hyperlinks permit users to simply click on a highlighted item that appears on their monitor and thereby retrieve information from (1) a different document on the same server, (2) a document on a different server anywhere on earth, or even (3) a different part of a current document. These hyperlinks are typically rendered by the browser in highlighted text on the user's screen. The HTML code must specify to the browser that a hyperlink is available. When directed, the browser transparently goes to the specified location and retrieves the requested piece of information.

To generate a hyperlink, five things must be included in the HTML code for the browser: (1) that a hyperlink is available, (2) what item is to be presented on the user's screen that, if clicked, will direct the browser to retrieve the information, (3) the type of server on which the information is located, (4) the pathname/address of the desired information, and (5) the format of the file to be retrieved.

Several types of servers are available. The initial portion of the pathname/address specifies the type of file server. The following list provides the most typical server types:

$$
\begin{array}{ll}
\text { Http:// } & \begin{array}{l}
\text { designates a World-Wide Web } \\
\text { server using hypertext transfer } \\
\text { protocol } \\
\text { designates a gopher server }
\end{array}
\end{array}
$$




\section{WAIS:// designates a Wide Area Informa- tion Service server \\ ftp:// designates a file transfer protocol service}

There are a wide variety of text, image, and movie formats. Therefore, it is necessary to use a file extension that specifies the file format to the browser. For example, if an image file is in "gif" format, then the file extension should be ".gif" to indicate that it is a gif image. Similarly, files that are saved as "X Bitmap" images should end in an ".xbm" file extension. The common file types and their extensions are:

$\begin{array}{ll}\text { Plain text } & \text { filename.txt } \\ \text { HTML document } & \text { filename.html } \\ \text { GIF image } & \text { filename.gif } \\ \text { TIFF image } & \text { filename.tiff } \\ \text { XBM image } & \text { filename.xbm } \\ \text { JPEG image } & \text { filename.jpeg } \\ \text { AIFF sound } & \text { filename.aiff } \\ \text { AU sound } & \text { filename.au } \\ \text { QuickTime movie } & \text { filename.mov } \\ \text { Mpeg movie } & \text { filename.mpeg }\end{array}$

Linking to different documents. In order to hyperlink from the current document to another document within the same directory on the same information server, the following format is used:

$<$ A HREF $=$ "filename. html" $>$ clickabletext $</$ A $>$

$\angle \mathrm{A}$ is the markup tag that specifies a hyperlink reference (i.e., a "clickable element"). The final text "clickabletext" is the text displayed by the browser on the user's screen. If the user clicks anywhere on that text, then the browser will display the file designated by the HREF $=" . .$. " Because the file is located within the same directory, a pathname/address is not needed.

To generate a link from the current document to another document in a different directory within the same server, or to a document located on any server anywhere on earth, the entire path name or uniform resource locator (URL) is typically required as a prefix. The URL, or standard pathname/address, functions just like a telephone number. An example HTML code is:

$<$ A HREF $=$ "http://jsucc.jsu.edu/
psychology/welcome.html">
Psychology Dept Welcome Page $</ A>$

In this case, the server uses hypertext transfer protocol (http://), the server name is "jsucc," and it is located in the educational domain named "jsu." The file "welcome.html" is located in the directory labeled psychol$o g y$. The clickable text displayed on the user's monitor is "Psychology Dept Welcome.Page."

Linking to an "external image." When a document contains many images, it can take quite a long time for a browser to download the entire file. A solution is to provide only miniature images in the primary display that are actually hyperlinks to the full size image. An ex- ternal image means that the image of interest must be opened as a separate document. Retrieving and displaying the larger external image is the same as retrieving any other information. To include a reference to an image as an external document, the format is:

$<$ A HREF = "pathname_address/filename.

filetype" $><$ IMG SRC="pathname_address/

filename.filetype" $</ A>$

An example HTML code is:

$$
\begin{aligned}
& \text { <A HREF ="http://jsucc.jsu.edu/psychology/gifs } \\
& \text { /pigeon.gif" ><IMG SRC="http://jsucc.jsu.edu } \\
& \text { /psychology/gifs/small.pigeon.gif" }></ A>
\end{aligned}
$$

The browser is notified that the code is the specification of a hyperlink (i.e., a clickable item) with $<A$. In place of the clickable text seen in the earlier example, $<$ IMG specifies that a clickable image is to be displayed. $\mathrm{SRC}=$ specifies that the location of that image follows, and "pathname_address/filename.filetype" is as described previously. Preceding this information, the object to be retrieved is specified as (HREF ="pathname_address/filename.filetype"). If the image displayed on the user's monitor is clicked, then the image located at the address following the hyperlink reference (HREF) is loaded as an external image.

Linking to specific sections within a document. A hyperlink to specific sections within a document provides the ability to jump forward or backward within the current document or to jump to specific sections of other documents. Each section of the document that will be used as a destination must be delimited and named as a potential destination address. The format is as follows:

$<$ A Name $=$ "targetname" $>$ targettext $</$ A $>$

The format in the HTML document that hyperlinks to that designated target name when the destination is in a different document is as follows:

$$
\begin{aligned}
& <\text { A HREF ="pathname_address/filename. } \\
& \text { filetype\#targetname" >clickabletext </A > }
\end{aligned}
$$

In this case, $<A$ specifies to the browser that a hyperlink is available; clickable text is the text that, if clicked, will load the target text; and HREF $=" .$. " specifies that the address of the text to be retrieved follows. "\#" specifies the name given to the subsection of that document or the target text. When the target text is in the same document as the hyperlink, then the format is as follows:

$$
<\mathrm{A} \text { HREF }=\text { \#targetname }>\text { clickabletext }</ \mathrm{A}>
$$

\section{EXAMPLE BROWSER DISPLAY OF AN HTML DOCUMENT}

There are two distinct environments created by the information flow on the World-Wide Web. One is from the point of view of the browser. In this case, the user is in- 


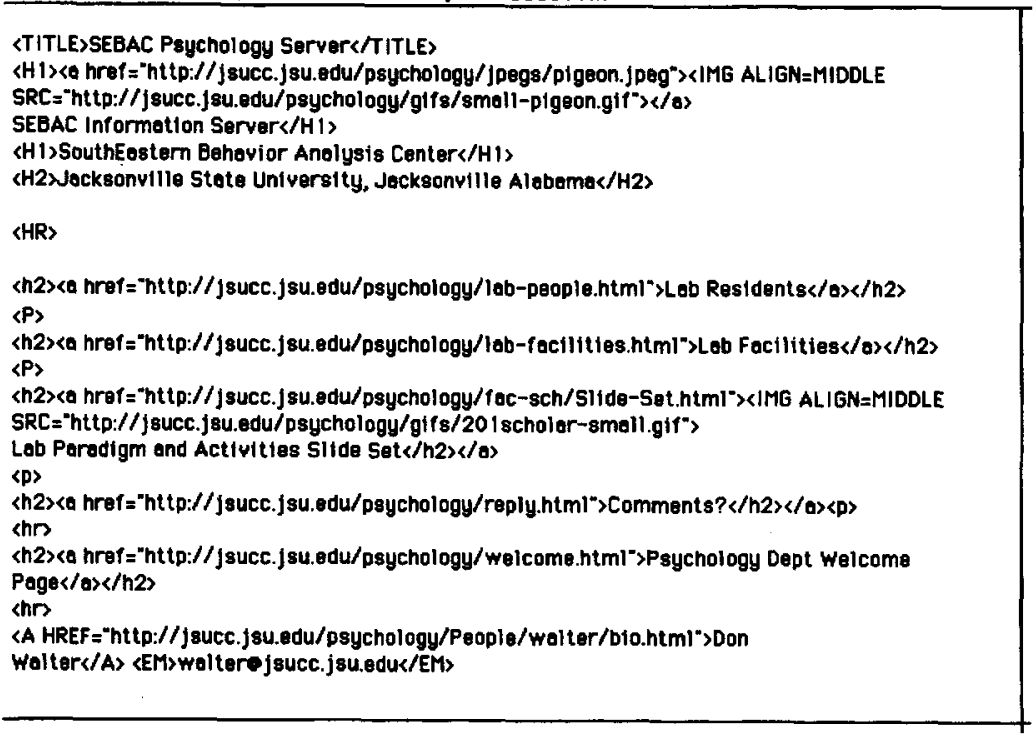

Figure 3. The actual HTML code that generated the SEBAC welcome page.

terested in exploring the information available on the Web. The second environment is from the point of view of the information provider. In this case, the user is interested in providing information to people on the net.

Browsers have user interfaces that are hierarchical. There is a root or base display page from which all others branch. This has led to two types of base pages. One type of page is optimized for users who are outbound to explore the net. In this case, the page is optimized for quickly invoking particular destinations ("hotlist") or searching tools such as "web crawler." This page is called a home page. A second type of base page is optimized for people who are inbound to an information server. In this case, the page is optimized for providing known information (most typically, the specific information provided by that server). For example, a map of where that particular site is located would be handy for incoming traffic but redundant for the people who were actually at that location and who were outbound. A páge optimized for inbound traffic is called a welcome page.

Figure 2 is an example of a welcome page (ours) as displayed by MacWeb. Figure 3 provides the first portion of the actual HTML code that produces that display. The display consists of a clickable miniature image (our logo), followed by a level one and a level two heading. This is followed by a horizontal rule, which separates the main heading from the body of the page. Below the horizontal rule are five lines of text that are hyperlinks. The third line is preceded by a clickable image that generates the same hyperlink as the text to its right. The final line on the page is the name and address of the author. This welcome page enables an inbound person to select the specific information they wish to obtain from our lab server. Clicking on "Lab Residents" retrieves a series of pictures of people who work in the lab. "Lab Facilities" provides a series of pictures of the lab. The image and text on the third line provide an image, which in turn links to five different slide sets that describe and explain, in detail, the lab's paradigm and activities. By clicking on different portions of the image, different sets of slides can be retrieved. The hyperlink "comments?" enables users to conveniently return messages to us through our e-mail system. The final hyperlink transfers the browser to the welcome page for the psychology department.

\section{Additional Information}

Further detailed information on HTML is available on the net by hyperlinks to the following URLs:

1. http://www.ncsa.uiuc.edu/General.Internet/ WWW/HTMLPrimer.html A Beginner's Guide to HTML

2. http://info.cern.ch/hypertext/WWW /MarkUp/Tags.html Peter Flynn's "How to write HTML"

3. http://kcgll .eng.ohio-state.edu/www/doc /htmldoc.html Ian Graham's guide to HTML

4. http://fire.clarkson.edu/doc/html/htut.html HTML Documents: A Mosaic Tutorial

5. http://www.utirc.utoronto.ca/HTMLdocs /NewHTML/htmlindex.html

(Manuscript received December 1, 1994; revision accepted for publication January 20,1995 .) 